\title{
Tecnologías digitales: análisis de planes de profesorado de Uruguay ${ }^{1}$
}

\section{Digital Technologies: Analysis of initial teacher training programmes in Uruguay}

ISSN 1510-2432 - ISSN 1688-9304 (en línea) - DOI: https://doi.org/10.18861/cied.2018.9.2.2858

Claudia Cabrera

Doctora en Educación y Master en Educación, Universidad ORT Uruguay. Licenciada en Ciencias Biológicas, Universidad de la República (Uruguay). Profesora de Biología, Instituto de Formación Docente de Florida. Docente de Biología y de Didáctica de Biología, Centro Regional de Profesores del Centro. Tutora de postgrados del Instituto de Educación, Universidad ORT Uruguay. Investigadora activa, Nivel Iniciación, Área Ciencias Sociales, Sistema Nacional de Investigadores (SNI).

\section{Ana Cabrera}

Diploma Internacional en Enseñanza con Tecnologías Digitales, University of Cambridge (Reino Unido). Diploma Superior en Educación y Nuevas Tecnologías, Facultad Latinoamericana de Ciencias Sociales (Argentina). Maestra de Educación Primaria. Secretaria Docente y Docente de Informática, Instituto de Formación Docente de Florida (Uruguay). Responsable científico, Proyecto PRAXIS: Formación pedagógico-didáctica en tecnologías y práctica docente Proyecto PRAXIS, Consejo de Formación en Educación (CFE) y Agencia Nacional de Investigación e Innovación (ANII).

Silvia Carámbula

Diploma Internacional en Enseñanza con Tecnologías Digitales. University of Cambridge (Reino Unido) - Consejo de Formación en Educación (CFE),Plan Ceibal.Diploma en Matemática mención enseñanza, Universidad de la República (Uruguay).Diploma en Evaluación de Aprendizajes, Universidad Católica del Uruguay. Profesora de Educación Media, Consejo de Educación Secundaria. Profesora de Matemática, Instituto de Formación Docente de Florida.

\section{Adriana Pérez}

Diploma Internacional en Enseñanza con Tecnologías Digitales, University of Cambridge (Reino Unido). Diploma en Evaluación de los Aprendizajes, Universidad Católica del Uruguay. Docente de Inglés, Consejo de Enseñanza Secundaria.

Marcela Pérez

Diploma Internacional en Enseñanza con Tecnologías Digitales, University of Cambridge (Reino Unido). Especialista en Didáctica de la Educación Superior,Universidad Claeh (Uruguay). Maestra de Educación Primaria, Instituto de Formación Docente de Florida (Uruguay). Docente de Inglés, Consejo de Enseñanza Secundaria.

Fecha de recibido: $28 / 08 / 2018$

Fecha de aceptado: 22/11/2018

\section{Resumen}

El siguiente artículo presenta un análisis histórico-comparativo de los últimos planes y programas de Formación Inicial Docente (FID) de profesorado de Educación Media en Uruguay en función del lugar asignado a las Tecnologías Digitales (TD). Cabe destacar que este trabajo se enmarca en un proyecto de investigación que busca indagar sobre las posibilidades, ventajas y limitaciones que la integración de TD aporta a la hora de reflexionar sobre las prácticas desarrolladas tanto por docentes en formación inicial como en formación en servicio. En esta instancia en particular se optó por un análisis de documentos y se trabajó con los planes de estudio 2005 y 2008 de la FID correspondientes a la carrera de 
Profesorado. En el caso del Plan 2005 se revisaron 151 programas de las asignaturas de los diferentes profesorados, en tanto que para 2008 se consultaron 238 programas. El estudio de los datos obtenidos revela, entre otros hallazgos, los siguientes: un relativo retroceso entre el plan actual (2008) y el anterior, ausencia de criterios comunes con respecto al lugar asignado a las TD en los programas de las asignaturas de los diferentes profesorados y referencias irregulares en cuanto a las conexiones entre la asignatura Informática y otras de la formación inicial. En el contexto aquí descrito se deja planteada la reflexión sobre los aspectos clave a considerar a la hora de tomar decisiones que posibiliten la inclusión genuina de las TD en la FID.

Palabras clave: formación docente, competencias digitales, cambio curricular, planes y programas, tecnologías digitales.

\section{Abstract}

The following article presents a historical and comparative analysis of the latest Programmes and syllabi in Initial Teacher Training (ITT) for high school teachers in Uruguay, focusing on the role assigned to digital technologies (DT). It is worth mentioning that this work has been carried out within the frame of a research project that seeks to find out the possibilities, advantages, and limitations of the integration of technology when reflecting upon the practices developed by both student teachers and in-service teachers. On this occasion, a documentary analysis has been chosen to examine the 2005 and 2008 ITT Programmes. With reference to the 2005 Programme, 151 syllabi corresponding to subjects in the different faculties were reviewed; whereas regarding the 2008 Programme, 238 curricular documents were analysed. Among other findings, the study of the data gathered evidences the following: a relative setback between the current Programme (2008) and the previous one; the absence of common criteria in relation to the place assigned to DT in the subject syllabi of the different faculties; and, irregular references in the Initial Teacher Training as for the connections between the subject Information and Communication Technology and the other subjects. In the context herein described, a deep reflection is made about the key aspects to be considered when making decisions that allow for the genuine inclusion of DT in the ITT.

Keywords: teacher training, digital competencies, curricular change, programmes and syllabi, digital technologies.

\section{Introducción}

Frente a los desafíos emergentes en la sociedad de la información y la comunicación, actualmente en Uruguay se debate el tránsito de la formación de docentes, de carácter terciario, hacia la Universidad de la Educación. En ese escenario resultan clave los aportes desde el ámbito internacional. Así, el informe de Unesco (2016, p.142) manifiesta lo siguiente: "la revisión de las propuestas de formación inicial de los docentes constituye una preocupación común instalada en la agenda de las políticas de las Tecnologías de la Información y la Comunicación (TIC)". Es en esta línea que la reflexión en torno al papel que desempeñan las Tecnologías Digitales (TD) en la formación de los futuros docentes cobra singular relevancia. Por ello, el análisis histórico de documentos curriculares tales como planes y programas es fundamental a la hora de identificar presencias y ausencias en relación con las TD, para desde una postura socio-crítica plantear sugerencias que se hagan eco de los vacíos existentes. Este artículo, que se enmarca en el Proyecto: "Praxis: Formación pedagógico-didáctica en tecnologías y práctica docente" (FSED_3_2016_1_133331), financiado por la Agencia Nacional de Investigación e Innovación de Uruguay (ANII) y el Consejo de Formación en Educación (CFE), busca entonces responder a interrogantes como las siguientes: ¿Qué presencias y ausencias se identifican en relación con las TD en los planes de formación de grado de profesorado de Uruguay en el período 2005-2017? 
¿Qué procesos, modelos de formación y desarrollo profesional se visualizan en dichas propuestas curriculares?

Estas preguntas guían el análisis de los planes de estudio, el cual se aborda en función de las categorías conceptuales estructuración y fundamentos. A partir de allí se proponen sugerencias ancladas en el contexto histórico nacional que sean de "valor teórico y práctico", según Yuni y Urbano (2006, p.76 - 77), al momento de pensar en la reforma.

\section{Antecedentes empíricos y teóricos Antecedentes empíricos}

En la actualidad existen múltiples trabajos de investigación referidos al conocimiento profesional de y mediado por TD, el cual se construye durante la formación inicial de los futuros docentes (FID) y se orienta a la conformación de su identidad como formadores. Tanto si el conocimiento profesional se concibe como conjunto de información, habilidades y valores (Montero, 2001) o como saberes prácticos y reflexión sobre la práctica (Marcelo, 1995), es posible considerar que el marco curricular o plan de estudio donde este conocimiento está inserto se constituye en el encuadre fundamental para su desarrollo. Tanto las presencias como las ausencias son elementos de análisis que dejan entrever fortalezas, debilidades y tensiones entre los distintos campos del saber que sustentan la formación de los futuros profesores. Autores como Gewerc \& Alonso (2017) realizan un análisis de antecedentes referidos a los contenidos de los planes de estudios en las propuestas de FID y, a partir del mismo, rescatan como principales las siguientes demandas:

“[...] mejorar los resultados académicos y el peso de la evaluación sistemática a sus espaldas; además de la necesaria atención a la diversidad en todas sus facetas y la mirada hacia lo social para cubrir y alertar cuestiones que, a veces, son desatendidas por la sociedad y las familias, etc". (Gewerc \& Alonso, 2017, p. 53).

En la misma línea, por un lado los hallazgos obtenidos por Gewerc \& Montero (2013, p.2) muestran en qué medida la incorporación de tecnología influye sobre "la actitud del profesorado frente a ellas, su formación y desarrollo profesional". Por otro lado el mismo estudio brinda aportes sustantivos en cuanto a cómo las decisiones en políticas de impulso a proyectos innovadores mediados por TD influyen en el profesorado y qué "procesos de formación y desarrollo profesional" son necesarios. Otro aporte que interesa es el de Tondeur et al. (2018) quienes señalan que se espera que las instituciones formadoras preparen a los futuros profesores para la incorporación de las TD en sus clases.

En este sentido, los hallazgos a los que arriban indican que cuanto más perciben los estudiantes de profesorado la ocurrencia de estrategias, más alto perciben su competencia en la incorporación de TIC tanto en los procesos de aprendizaje como en la mejora de su práctica docente. En lo que refiere a Latinoamérica, es relevante señalar el estudio realizado por Robalino y Körner (2005) por medio del análisis de experiencias de formación de docentes utilizando TD en Bolivia, Chile, Colombia, Ecuador, México, Panamá, Paraguay y Perú.

Además de considerar la incorporación de TD como "imperativo" en la FID, los autores hacen especial énfasis en la necesidad de que esta práctica se establezca como "un soporte transversal y constituyente del currículo" (p. 22). Si el foco se centra en la experiencia de Uruguay en la última década, es fundamental considerar las recomendaciones de Vaillant (2013): 
"Hay que pensar en los diversos aspectos que hacen al problema; desde quiénes son los estudiantes que hoy ingresan a las universidades e institutos de formación, pasando por los planes de estudio, las estrategias pedagógicas y los formadores. Y es en ese contexto que se inscribe la incorporación de las TIC, que no puede ser considerada aisladamente ni a partir de aspectos puntuales, sino como un componente más de las políticas docentes". Vaillant, D. (2013, p. 45)

Las diferentes investigaciones relevadas dan cuenta de que, en general, el docente y su formación inicial se constituyen en agentes clave para la inserción de TD en la educación. En este sentido Silva Quiroz, Miranda \& Gisbert (2016) indagan sobre la incorporación de TD en los planes de formación docente de la región y del país, y destacan que la inmersión tecnológica en los sistemas educativos es de larga data, tanto en América Latina como en Uruguay (Silva Quiroz, Miranda \& Gisbert, 2016). En oposición a lo expuesto, un estudio realizado recientemente por Rodríguez Zidán et al. (2018) presenta como hallazgo la paradoja que se evidencia entre el amplio acceso a las TD por parte de los estudiantes de formación docente de Uruguay y el escaso uso de TD que se constata en el ámbito de la FID en los diferentes núcleos formativos: asignaturas específicas, del núcleo de formación profesional común y didáctica práctica docente. En estrecha relación con lo mencionado interesa el aporte de Ottenbreit-Leftwich et al. (2012) quienes afirman que los docentes no aprecian que las habilidades tecnológicas específicas que adquirieron en su formación inicial sean personalmente significativas para sus prácticas. La misma idea es apoyada por Gülbahar (2008), quien expresa que a pesar de que existe disposición para el uso de TD por parte de los docentes esto no es lo que se favorece desde los programas instruccionales.

Como se mencionara, el presente artículo intenta establecer cómo y en qué medida la oferta de formación en TD, presente en los Planes 2005 y 2008 de Formación de Profesores de educación media en Uruguay, ha contribuido o contribuye al desarrollo del conocimiento profesional en consonancia con las exigencias formativas actuales y las perspectivas a considerar hacia el cambio curricular próximo. En este escenario resulta relevante considerar el Marco Europeo de Competencias Digitales para los Ciudadanos (Ferrari, 2013) del Instituto de Prospectiva Tecnológica de la Unión Europea, que pone en foco las cinco áreas de competencias digitales docentes (CDD): Información, Comunicación, Creación de contenidos, Seguridad y Resolución de Problemas. Asimismo, es necesario tener presente los planteos de Gewerc \& Montero (2015) en relación con los contenidos, los procesos de formación y el conocimiento profesional necesario para establecer "intersecciones entre el conocimiento pedagógico, del contenido y de la tecnología en la formación inicial del profesorado" (p.34). Es en esta línea que se aspira a aportar a la reflexión sobre lo que deben aprender los futuros docentes para dar respuesta profesional a las situaciones emergentes en un mundo complejo y caracterizado por el cambio tecnológico permanente.

\section{Antecedentes teóricos}

En el siguiente apartado se presentarán las ideas teóricas clave que dan fundamento al presente artículo.

\section{Formación del profesorado}

La formación del profesorado no debe estar ajena a los cambios que suceden en la sociedad del siglo XXI. Esta debe estar en sintonía con los mismos y preparar a los futuros docentes y docentes noveles para que sean capaces de incluir las TD de forma pertinente, crítica y reflexiva en sus prácticas. 
Según Belando-Montoro (2014) la formación permanente del profesorado debe incluir la competencia en el uso de las TD, y dicha formación debe desarrollarse de forma transversal. En este sentido surgen interrogantes tales como: ¿Cómo lograr un uso reflexivo de las TD? ¿Qué habilidades deben estar presentes en el profesorado? ¿Cómo se refleja e influye en la evaluación? Se trata de una "nueva ecología del aula universitaria en la cual las TD pasan a tener un rol importante" en "la sociedad de la pantalla" según Levis (2016).

Es innegable que las múltiples posibilidades que brindan las TD hoy en día no están exentas de exigencias. Frente a las mismas, Avello et al. (2014) se preguntan si los docentes están preparados para enfrentarlas y, aún más, si lo que se está haciendo asegura una formación docente apropiada.

Asimismo, en su informe 2013 la Unesco señala que las instituciones de formación docente o bien asumen un rol protagónico en la transformación de la educación o se quedan rezagadas en el continuo desarrollo del cambio tecnológico. A este respecto es vasta la literatura existente que, a la vez que señala la superficialidad en la incorporación de TD a la docencia universitaria -donde se conservan prácticas pedagógicas tradicionales y carentes de creatividad- se enfatiza en la necesidad de asumir las prácticas mediadas por TD como puentes hacia la creación innovadora (Díaz Barriga, 2010; Gros, 2000; Quiroga, 2011; Gros y Suárez Guerrero, 2016; Cobo, 2016). Para generar cambios en las creencias y en las prácticas que sean sostenibles como señala Lion (2015), las propuestas educativas deben poder adecuarse a un contexto de cambio permanente, a una formación continua del profesorado en escenarios de "comunidades o redes" (Fullan, 2002). Tal y como remarca Lion (2015), "las tecnologías no generan un cambio per se, sino que se incorporan en tramas institucionales con su cultura organizacional" (p. 12).

Dado que en los planes de estudio de profesorado en general no se especifica claramente cómo integrar las TD para apoyar el aprendizaje, estamos de acuerdo con lo sugerido por Unesco (2004) con respecto a quienes definen dichos planes en que es necesario optimizar la incorporación de dichas tecnologías a lo largo de toda la formación y “diseñar experiencias formativas prácticas para los futuros docentes" (Unesco, 2004, p.65).

En cuanto a los programas, este informe propone que "los cursos de formación docente deben brindar a sus estudiantes experiencias ricas en tecnología en todas las áreas y aspectos del programa" (Unesco, 2004, p.65).

\section{Desarrollo de CDD}

Ante la necesidad de mejorar el desempeño profesional docente, y por ende los aprendizajes de los estudiantes, cabe reflexionar sobre el desarrollo de competencias, las que se definen como la capacidad de "movilizar varios recursos cognitivos para hacer frente a un tipo de situaciones" (Perrenoud, 2007, p. 8).

Dado que el foco de interés aquí se centrará en la Competencia Digital (CD) interesa lo mencionado por Krumsvik (2007) -citado por Ottestad, Gudmundsdottir y Kelentric (2014)- cuando define a la CDD como "la habilidad docente de usar las TIC con una buena comprensión pedagógico-didáctica y ser consciente de cómo esto impacta en las estrategias de aprendizaje y la formación educativa de los estudiantes" (p.68, traducción propia).

Indudablemente, el concepto de CDD ha sido abordado con recurrencia en las investigaciones referidas a Tecnología Educativa. En este estudio en particular se identifica la siguiente definición como vertebradora de las decisiones a tomar: 
“La CDD está constituida por un conjunto de conocimientos, destrezas y actitudes que permiten, por una parte, usar de modo eficiente en el ámbito educativo los diferentes dispositivos tecnológicos vinculados a la enseñanza; las aplicaciones y servicios digitales de gestión y comunicación: así como los contenidos digitales vinculados con las áreas y materias curriculares. Por otro lado, permiten utilizar las estrategias adecuadas para promover en el alumnado el desarrollo de su propia competencia digital". (Valverde, 2015, p. 221).

Se piensa que el análisis de las CDD del profesorado universitario es fundamental ya que aborda sus tres áreas de desempeño: docencia, investigación y gestión. Durán, Gutiérrez \& Prendes (2016) proponen un modelo de CDD actualizado para docentes universitarios según las dimensiones a saber: Las básicas como ciudadano: tecnológica, comunicativa, informacional, multimedia, de seguridad y de resolución de problemas;

Las específicas de la tarea docente: gestión de la docencia apoyada en TD, evaluación del aprendizaje con TD, potencial didáctico de las TD, formación y TD y facilitación del aprendizaje y creatividad del alumno con TD; y

Las concretas del ámbito profesional: la investigación e innovación pedagógica con y para el uso de las TD, y la publicación y difusión de material en la Web.

Dentro del contexto latinoamericano en general y en la realidad uruguaya en particular deben destacarse los hallazgos realizados por Silva Quiroz et al. (2016) quienes proponen una matriz de indicadores agrupados en cuatro dimensiones:

1) Didáctica, curricular y metodológica;

2) Planificación, organización y gestión de recursos tecnológicos;

3) Aspectos éticos, legales y seguridad; y

4) Desarrollo personal y profesional.

El estudio antes mencionado amplía pues la comprensión de la realidad de la FID en Uruguay, da cuenta del estado actual de desarrollo de las CDD y recomienda lo siguiente: "Los estándares e indicadores en CDD existentes, [...] requieren planes de implementación en la formación de los futuros docentes, reflejados en las mallas curriculares formativas de asignaturas y/o a través de un uso transversal de la tecnología en las diferentes dimensiones del perfil del docente en formación". (Silva Quiroz et al., 2016, p.66).

El presente artículo toma en cuenta esta sugerencia y utiliza el Marco Común del Instituto Nacional de Tecnologías Educativas y de Formación del Profesorado (INTEF, 2017), Marco Común de Competencia Digital Docente - Setiembre 2017 (MCCDD) como guía en la definición de una de las categorías consideradas para el análisis de los Planes 2005 y 2008. Cabe señalar que el MCCDD tiene su antecedente en el Proyecto DIGCOMP (Ferrari, 2013). La propuesta más reciente tiene como objetivos: contribuir a que los profesores conozcan, ayuden a desarrollar y evalúen las CD de sus estudiantes; facilitar descriptores que sirvan como referencia y contribuyan a establecer los requisitos mínimos de CD con los que deben contar los formadores para incorporar TD a sus prácticas; y propiciar cambios 
metodológicos tanto en el uso de TD como en los procesos de aprendizaje y de enseñanza en general. A los efectos de relevar en qué medida las últimas propuestas curriculares nacionales reflejan o no el estímulo al desarrollo de las CDD en la FID, se consideraron cinco áreas y sus correspondientes competencias (Figura 1).

\begin{tabular}{|c|c|}
\hline ÁREA DE COMPETENCIA & COMPETENCIAS RELACIONADAS \\
\hline $\begin{array}{l}\text { Área } 1 . \\
\text { Información y alfabetización } \\
\text { informacional }\end{array}$ & $\begin{array}{l}\text { 1.1 Navegación, búsqueda y filtrado de información } \\
\text { 1.2 Evaluación de información } \\
\text { 1.3 Almacenamiento y recuperación de información }\end{array}$ \\
\hline $\begin{array}{l}\text { Área } 2 . \\
\text { Comunicación y colaboración }\end{array}$ & $\begin{array}{l}\text { 2.1. Interacción mediante las tecnologías digitales } \\
\text { 2.2. Compartir información y contenidos digitales } \\
\text { 2.3. Participación ciudadana en línea } \\
\text { 2.4. Colaboración mediante canales digitales } \\
\text { 2.5. Netiqueta } \\
\text { 2.6. Gestión de la identidad digital }\end{array}$ \\
\hline $\begin{array}{l}\text { Área } 3 . \\
\text { Creación de contenidos digitales }\end{array}$ & $\begin{array}{l}\text { 3.1. Desarrollo de contenidos digitales } \\
\text { 3.2. Integración y reelaboración de contenidos digitales } \\
\text { 3.3. Derechos de autor y licencias } \\
\text { 3.4. Programación }\end{array}$ \\
\hline $\begin{array}{l}\text { Área } 4 . \\
\text { Seguridad }\end{array}$ & $\begin{array}{l}\text { 4.1. Protección de dispositivos } \\
\text { 4.2. Protección de datos personales e identidad digital } \\
\text { 4.3. Protección de la salud } \\
\text { 4.4. Protección del entorno }\end{array}$ \\
\hline $\begin{array}{l}\text { Área } 5 . \\
\text { Resolución de problemas }\end{array}$ & $\begin{array}{l}\text { 5.1. Resolución de problemas técnicos } \\
\text { 5.2. Identificación de necesidades y respuestas } \\
\text { tecnológicas } \\
\text { 5.3. Innovación y uso de la tecnología digital de forma } \\
\text { creativa } \\
\text { 5.4. Identificación de lagunas en la competencia digital }\end{array}$ \\
\hline
\end{tabular}

Figura 1. Elaboración propia a partir de información aportada por INTEF. Marco Común de Competencia Digital Docente - Setiembre 2017, p.14.

Corresponde mencionar que existen posicionamientos que critican lo planteado por INTEF (2017), basados en que solo se considera al docente y a su trabajo a nivel de aula sin aludir, por ejemplo, al impacto que puede tener la gestión de tecnologías sobre el contexto social de los educandos. En esa línea se encuentra el modelo presentado por Castañeda, Esteve y Adell (2018) que amplía lo propuesto por otros autores e incorpora nuevas categorías de análisis (Figura 2).

Si bien se comparte plenamente el Modelo de competencia docente integral (Castañeda, Esteve y Adell, 2018, p.13), se estima que resulta demasiado amplio para analizar las propuestas programáticas de la FID de Uruguay y es por ello que aunque el modelo propuesto por INTEF (2017) sea restrictivo resulta válido para guiar dicho análisis. 


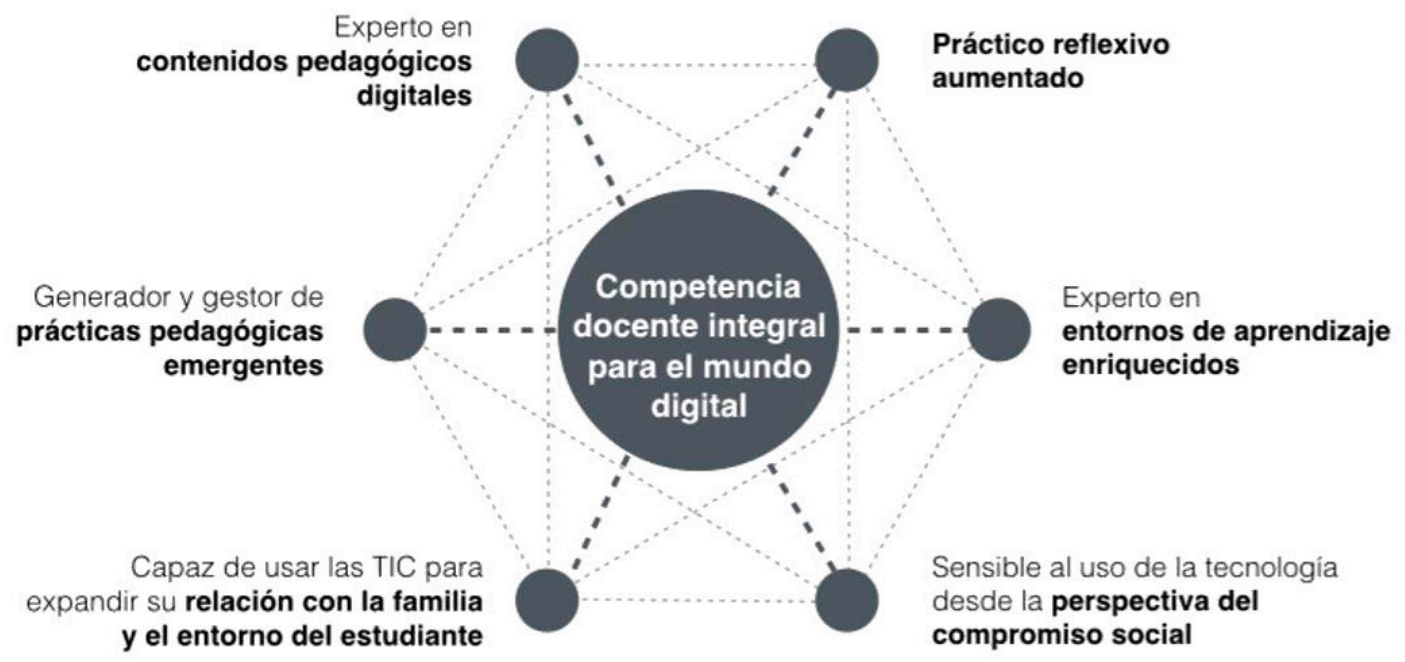

Figura 2. Modelo de competencia docente integral en el mundo digital.

Fuente: Castañeda, Esteve y Adell (2018, p.13)

\section{Modelo TPACK (Technology, Pedagogy and Content Knowledge, traducido como Tecnológico, Pedagógico y Disciplinar)}

La investigación que da lugar a este artículo se encuentra en proceso de desarrollo y se propone indagar cómo se integran las TD a las prácticas de manera reflexiva. Es por ello que al explorar los Planes de Formación Docente se pretende aportar a la reflexión sobre las presencias y ausencias en lo que refiere a las CDD así como a las intersecciones entre el contenido disciplinar, el pedagógico y el tecnológico.

No cabe duda de que los centros educativos deben desarrollar estrategias pertinentes para favorecer la adquisición de competencias clave en el manejo de las TD. Sin embargo, “¿Hasta qué punto los actuales planes de estudio están respondiendo a estas nuevas demandas? ¿Cuál es la formación tecnológica, didáctica y disciplinar del profesorado?" (Esteve y Parejo, 2014, p. 188).

En lo referente a la segunda interrogante, Esteve y Gisbert (2011, p. 64) señalan que “como docentes, sólo a partir de una adecuada combinación de conocimiento tecnológico, disciplinar y didáctico-pedagógico podremos hacer servir todas las potencialidades de las TICs para facilitar procesos de aprendizaje activos, participativos y centrados en el alumno". Esta visión es congruente con el planteo de Cejas, Navío \& Barroso (2016, p. 105) que sostienen que "el modelo TPACK (conocimiento pedagógico y tecnológico del contenido, desarrollado por Koehler \& Mishra) presenta una vía interesante para que los profesores puedan integrar tecnología, pedagogía y conocimiento disciplinar en su función docente" (Figura 3).

No solo se trata de integrar estos componentes sino también de tener capacidad de flexibilidad y adaptabilidad frente a los cambios constantes que se dan en la sociedad. Es así que resulta clave que el docente maneje el modelo TPACK y los desafíos que el mismo conlleva. 


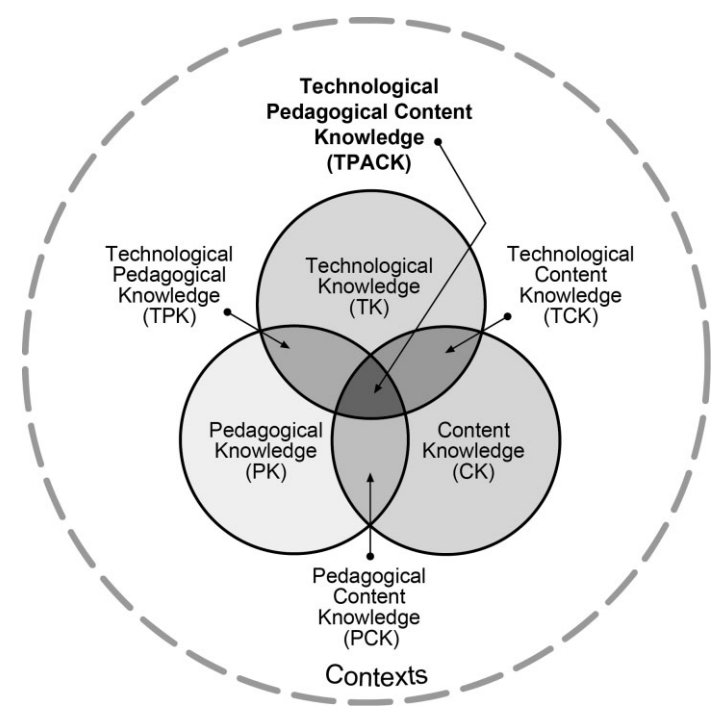

Figura 3. Modelo TPACK. Imagen reproducida con permiso del autor. (c) 2012 by tpack.org

Una enseñanza significativa implica pues alcanzar y mantener el equilibrio en el manejo de todos los componentes del TPACK. Es dicho equilibrio el que habilita a una ecología de aula sin fronteras donde las tecnologías digitales puedan integrarse completamente, y donde el rol del docente sea de "asesor formativo" según Cejas, Navío \& Barroso (2016).

\section{Metodología}

El foco de este artículo es conocer el marco curricular de la formación inicial de profesores. En particular interesa conocer cuáles son las líneas curriculares referidas a TD que rigen el proceso de formación actual correspondiente al Plan 2008. También es relevante aproximarse al proceso histórico del que ese plan forma parte. Para ello resulta sustancial conocer el Plan 2005 como antecedente inmediato en la formación de docentes en los Centros Regionales de Profesores así como la actual proyección hacia una reformulación curricular.

Para llevar adelante el estudio de los programas se recurrió a un abordaje metodológico mixto (Hernández Sampieri, 2014) basado en el análisis de documentos. Se realizó un análisis exhaustivo que consistió en relevar los fundamentos de los planes 2005 y 2008 , así como los programas de todas las asignaturas de los diferentes profesorados. El trabajo incluyó el análisis de 151 programas de asignaturas correspondientes al Plan 2005 y 238 programas de asignaturas del Plan 2008. También fueron objeto de estudio los documentos oficiales correspondientes a la reformulación curricular. Corresponde mencionar que en el caso del Plan 2005 no fue fácil acceder a los programas oficiales y en algunos casos lo publicado en el sitio Web del CFE corresponde a borradores de trabajo, por lo cual fue necesario recurrir a la base de información entregada en formato digital a las direcciones de los centros. Una vez que se accedió a los diferentes programas se inició el proceso de recolección de datos a partir de la búsqueda utilizando palabras clave. Dado que los programas fueron diseñados hace varios años -2004 y 2007 respectivamente para cada plan- se realizó una lista de palabras clave para ampliar el espectro de búsqueda y así identificar cualquier referencia vinculada a TD, ya que con esa denominación exacta los resultados fueron nulos. Los términos que orientaron el relevamiento fueron: software, tecnologías, TIC, programas, aplicaciones, sensores, digital, NTIC (Nuevas Tecnologías de la Información y la Comunicación), informática. 
Para la sistematización de la información se abordó el análisis en dos niveles. Por un lado, se consideraron los fundamentos de los Planes 2005 y 2008 y los fundamentos de la reformulación curricular 2017. Por otro lado se recurrió al análisis de los programas de las asignaturas de los diferentes profesorados. Interesó especialmente la propuesta curricular referida a la asignatura Informática, por entender que es un espacio directamente vinculado a las TD. En el caso de los programas del Plan 2008 (plan vigente en la actualidad) interesó identificar en qué sección de los programas (Fundamentación, Objetivos, Contenidos, Metodología, Evaluación, Bibliografía) se localiza la alusión a TD.

El proceso de categorización fue mixto. Aunque la mayoría de las categorías son, a priori, basadas fundamentalmente en las propuestas por Gewerc (2017), otras surgen de lo empírico. Algunos ejemplos de las primeras son: contenidos, relaciones con otras asignaturas, jerarquización (periodicidad y tiempo), entre otras. Como ejemplo de las empíricas se encuentran las categorías que incluyen componentes integrados de otras categorías, tal como es el caso de aquellos contenidos que tienen tanto de alusión al uso instrumental como de aplicación a la práctica de las TD.

Una vez identificadas las categorías se llevó a cabo la construcción de matrices de datos que permitió la sistematización de los mismos para la correspondiente elaboración de teoría.

\section{Resultados}

\section{Cambio de planes en la FID. ¿Avances o retrocesos en el desarrollo de las competencias digitales?}

La comparación entre planes se realizó en base a tres indicadores:

(1) Las características de la asignatura Informática;

(2) La cantidad de alusiones a TD en otras asignaturas;

(3) La fundamentación de cada plan.

Por una parte, en lo que respecta a las características de la asignatura Informática es posible constatar que, además de dedicarse una mayor carga horaria al abordaje de la misma en el Plan 2005, es en dicho plan en el que se encuentra, de forma explícita, la relevancia de establecer conexiones con otras asignaturas de la FID. Un ejemplo claro se halla en el presente pasaje del Programa de Informática: “Incentivar la articulación entre Informática y el área de Ciencias de la Educación del currículo de 2 do - especialmente con la Práctica Docente" (Programa de Informática $2^{\circ}$, p. 1). Por otra parte, aun cuando el Plan 2008 deja entrever que deben establecerse dichos vínculos no lo marca con igual nivel de explicitación, lo que puede apreciarse en la siguiente cita: "Búsqueda de información en Internet. [...] Aplicación a distintas disciplinas y áreas de conocimiento". (Programa de Informática Plan 2008, p.2.)

\begin{tabular}{|c|c|c|c|}
\hline & & PLAN 2005 & PLAN 2008 \\
\hline \multirow[t]{4}{*}{$\begin{array}{l}\text { Asignatura } \\
\text { Informática }\end{array}$} & $\begin{array}{l}\text { Cantidad de horas } \\
\text { semanales }\end{array}$ & 2 por nivel & 3 por nivel \\
\hline & $\begin{array}{l}\text { Frecuencia durante la } \\
\text { carrera }\end{array}$ & $\begin{array}{l}\text { En primero, } \\
\text { segundo y tercero }\end{array}$ & Solo en tercero \\
\hline & $\begin{array}{l}\text { Horas semanales totales } \\
\text { en el plan }\end{array}$ & 6 & 3 \\
\hline & $\begin{array}{l}\text { Relación con otras } \\
\text { asignaturas }\end{array}$ & Explícito & Implícito \\
\hline
\end{tabular}

Figura 4. Cuadro comparativo de las características de la asignatura Informática en Plan 2005 y Plan 2008. Fuente: Elaboración propia. 
Si bien la asignatura Informática es la que debería estar directamente relacionada con el desarrollo de la CD, también interesa visualizar cómo el resto de las asignaturas aportan al desarrollo de dicha competencia. La referenciación a las TD desde diferentes asignaturas aportaría a lo que Robalino y Körner (2005) denominan un abordaje transversal de las mismas en la formación de los docentes. A este respecto, lo que emerge de la comparación entre los planes es un comportamiento muy variado de acuerdo al tipo de asignaturas y al área del conocimiento que se trate. En el caso de las ciencias de la educación, estas pasan de tener un 16\% de presencia en Plan 2005 a 0\% en el plan 2008. En lo que refiere a las asignaturas específicas de cada área de profesorado (Ciencias Sociales-CCSS, Ciencias de la Naturaleza-CCNN, Matemática y Lengua), no se mantiene la misma lógica. Mientras que en CCNN el porcentaje de alusión a TD en las didácticas disminuye en el Plan 2008 con respecto al Plan 2005, en las otras asignaturas específicas se aprecia un claro aumento: pasa de $17 \%$ en 2005 a 30\% en 2008. En lo que respecta a Didáctica en las CCSS y Lengua, llama la atención que en el primer caso pasa de un 22\% en 2005 a 0\% en 2008, situación que se invierte para Lengua, donde la alusión a TD en el Plan 2005 pasa de estar ausente a tener una presencia de un 19\% en 2008. Estos cambios aleatorios parecen evidenciar que al momento de cambiar de plan no existieron criterios comunes en relación con el lugar conferido a las TD en los programas de las asignaturas de los diferentes profesorados.

\begin{tabular}{|c|c|c|c|c|}
\hline \multicolumn{3}{|c|}{ Referencia a TD en los programas } & Plan 2005 & Plan 2008 \\
\hline \multirow[t]{8}{*}{ Específicas } & \multirow{4}{*}{ Otras específicas } & $\begin{array}{l}\text { Ciencias de la Naturaleza y } \\
\text { Matemática }\end{array}$ & $17 \%$ & $30 \%$ \\
\hline & & Ciencias Sociales & $27 \%$ & $12 \%$ \\
\hline & & Lengua & $0 \%$ & $0 \%$ \\
\hline & & Total & $15 \%$ & $14 \%$ \\
\hline & \multirow{4}{*}{$\begin{array}{l}\text { Didáctica - } \\
\text { Práctica Docente }\end{array}$} & $\begin{array}{l}\text { Ciencias de la Naturaleza y } \\
\text { Matemática }\end{array}$ & $58 \%$ & $38 \%$ \\
\hline & & Ciencias Sociales & $22 \%$ & $0 \%$ \\
\hline & & Lengua & $0 \%$ & $19 \%$ \\
\hline & & Total & $33 \%$ & $20 \%$ \\
\hline \multicolumn{3}{|c|}{ Ciencias de la educación } & $16 \%$ & $0 \%$ \\
\hline
\end{tabular}

Figura 5. Cuadro comparativo de las referencias a TD en los programas del Plan 2005 y Plan 2008. Fuente: Elaboración propia.

Además de considerar los contenidos presentes en las asignaturas incluidas en cada plan interesa analizar cómo se posiciona cada propuesta desde su fundamentación con respecto a las TD y su relevancia en la FID. Dado que en la actualidad la FID se encuentra en pleno proceso de reformulación, interesa además considerar cómo se está pensando el cambio en lo que respecta al desarrollo de la CD. Para ello se tomó como referencia el documento denominado Nueva propuesta curricular hacia el Nuevo Plan 2017. Fundamentos del Plan 2017. Al comparar las tres propuestas formativas se aprecia que mientras que desde su fundamentación el plan vigente (Plan 2008) alude de manera muy general a la tecnología sin nombrar ningún sinónimo de TD, el Plan 2005 menciona su relevancia y la relaciona fundamentalmente con la formación instrumental refiriendo además a la necesidad de 
establecer nexos con el desempeño profesional. En lo que respecta a los lineamientos del nuevo plan, se hace referencia de forma muy general a dichas tecnologías, aunque es válido aclarar que la expresión: "uso creativo y con sentido de las tecnologías digitales" da cuenta de un corrimiento de una perspectiva meramente instrumental de las mismas. Lo aquí mencionado podría interpretarse como una intención de aproximación a paradigmas más reflexivos para la formación de los futuros educadores. Aun así resulta necesario que los cambios que se realicen a nivel curricular realmente apunten a promover transformaciones en las prácticas de enseñanza (Ottenbreit-Leftwich et al., 2012).

\section{Aproximación de la propuesta curricular 2008 al modelo TPACK}

En el presente estudio se analizaron los contenidos referidos a TD presentes en los programas de las asignaturas de 11 profesorados del Plan 2008. Una vez identificados dichos contenidos, se los categorizó de acuerdo con la relación que se establecía entre el contenido tecnológico y el conocimiento disciplinar y didáctico-pedagógico, situados aquellos en la intersección de los tres campos del conocimiento. El interés por encontrar evidencias en los programas que hagan explícita la relación entre lo disciplinar, lo didáctico y lo tecnológico se basa en la idea de varios autores (Esteve y Gisbert, 2011; Cejas, Navío \& Barroso, 2016; entre otros) que conciben al modelo TPACK como una oportunidad de aprovechar al máximo las potencialidades de las TD. Cabe mencionar que la sola presencia de sugerencias de conexiones entre los diferentes tipos de contenido en los programas no garantiza que las mismas se pongan en práctica en las aulas. Sin embargo, dicha alusión refleja el interés por la promoción de abordajes más integrados.

A partir del análisis realizado en este estudio surge la identificación de contenidos que evidencian vinculación entre lo tecnológico y lo disciplinar, otros que muestran afinidad con lo didáctico pedagógico y finalmente los que marcan la relación entre los tres tipos de contenidos: tecnológico, didáctico y disciplinar. En la Figura $N^{\circ} 6$ se visualizan algunos ejemplos que permiten comprender el alcance de cada grupo.

\begin{tabular}{|c|c|c|c|}
\hline & \multicolumn{3}{|l|}{ Tipo de contenidos } \\
\hline & $\begin{array}{l}\text { Tecnológico - } \\
\text { Disciplinar }\end{array}$ & $\begin{array}{l}\text { Tecnológico - } \\
\text { Didáctico }\end{array}$ & $\begin{array}{l}\text { Tecnológico - Disciplinar- } \\
\text { Didáctico }\end{array}$ \\
\hline $\begin{array}{l}\text { Conceptualización } \\
\text { (Cejas, Navío y } \\
\text { Barroso, 2016. p. } \\
\text { 113) }\end{array}$ & $\begin{array}{l}\text { "Conocimiento sobre } \\
\text { cómo la tecnología } \\
\text { puede utilizarse } \\
\text { para representar } \\
\text { la materia a utilizar } \\
\text { y desarrollar la } \\
\text { competencia } \\
\text { disciplinar". }\end{array}$ & $\begin{array}{l}\text { "Competencias que } \\
\text { incluyen aspectos } \\
\text { tecnológicos y } \\
\text { pedagógicos". }\end{array}$ & $\begin{array}{l}\text { “Conocimientos sobre } \\
\text { cómo usar la tecnología } \\
\text { más adecuada en un } \\
\text { marco pedagógico } \\
\text { para la impartición de } \\
\text { determinada materia". }\end{array}$ \\
\hline $\begin{array}{l}\text { Evidencias que } \\
\text { ejemplifican }\end{array}$ & $\begin{array}{l}\text { “Visualización a } \\
\text { través del Google } \\
\text { Earth. (Programa } \\
\text { de la asignatura } \\
\text { Elementos de } \\
\text { Astronomía del } \\
\text { Profesorado de } \\
\text { Geografía", p.2) }\end{array}$ & $\begin{array}{l}\text { "Recursos } \\
\text { didácticos: uso del } \\
\text { libro del texto, uso } \\
\text { del ordenador, uso } \\
\text { de la calculadora, } \\
\text { los softwares } \\
\text { educativos y } \\
\text { applets". (Programa } \\
\text { de la asignatura } \\
\text { Didáctica III del } \\
\text { Profesorado de } \\
\text { Matemática, p.4) }\end{array}$ & $\begin{array}{l}\text { “Empleo de nuevas } \\
\text { tecnologías, se procurará } \\
\text { introducir al futuro } \\
\text { profesor en Ciencias } \\
\text { Biológicas, en el } \\
\text { empleo de éstas en la } \\
\text { educación, tanto para el } \\
\text { estudio de contenidos } \\
\text { temáticos, como para su } \\
\text { transposición didáctica". } \\
\text { (Programa de la asignatura } \\
\text { Biofisica del Profesorado } \\
\text { de Biología, p.4) }\end{array}$ \\
\hline
\end{tabular}

Figura $\mathbf{N}^{\circ}$ 6. Conceptualización y ejemplos de los diferentes tipos de contenidos propuestos por el modelo TPACK.

Fuente: Elaboración propia. 
Al momento de cuantificar la aparición de cada tipo es posible apreciar (Figura $N^{\circ} 7$ ) que poco más de un tercio de las referencias se identifican con la integración de los tres tipos de conocimiento (39\%) y que le sigue, en porcentaje, el tipo que vincula lo tecnológico con la formación disciplinar (36\%).

Tipo de conocimiento al que se hace referencia cuando se alude a TD

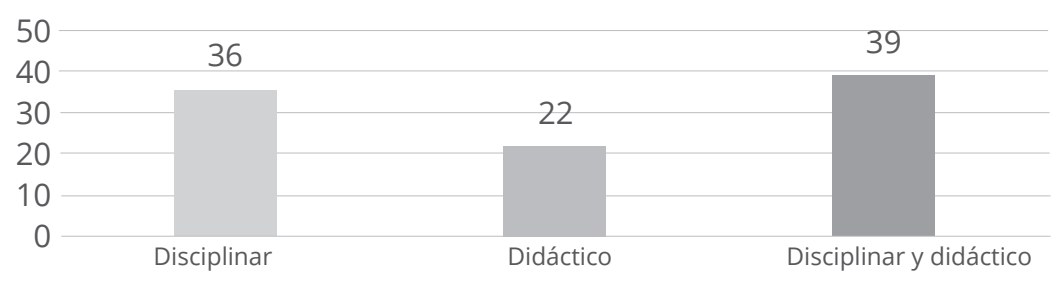

Figura $\mathbf{N}^{\circ}$ 7. Tipo de conocimiento al que hacen referencia cuando aluden a TD.

Fuente: Elaboración propia.

Si se consideran los aportes de Robalino y Körner (2005) y de Tondeur et al. (2018) que sostienen que es fundamental que los estudiantes de profesorado reciban, en su formación inicial, orientaciones que vinculen lo tecnológico a lo didáctico, se encuentra que, de las escasas alusiones a lo tecnológico en los programas de las diferentes asignaturas (14\% en otras específicas y $20 \%$ en didácticas), si se suma el $22 \%$ que alude a lo didáctico y el $39 \%$ que alude a lo didáctico y a lo disciplinar, solo un $61 \%$ contempla la vinculación explícita entre ambas formas de conocimiento (conocimiento tecnológico y conocimiento didáctico).

\section{Presencia del conocimiento tecnológico en las diferentes áreas del conocimiento}

Como parte del análisis de los programas se realizó la comparación de la frecuencia con la que aparecen alusiones a TD en las diferentes áreas del conocimiento, y a su vez se consideró la variable tipo de asignatura distinguiendo entre lo relevado en didáctica y lo relevado en las otras asignaturas específicas. Es el área de CCNN y Matemática la que muestra mayores referencias a TD en los programas de sus asignaturas, tanto en didáctica como en otras específicas (Figura $\mathrm{N}^{\circ} 8$ ).

Otro aspecto que merece ser analizado es que la referencia a TD en las CCSS se da en asignaturas específicas y fundamentalmente en el profesorado de Geografía seguido por el profesorado de Derecho. Sin embargo, los profesorados de Historia y Sociología no hacen alusión alguna a las TD. En el área de Lengua ocurre lo contrario: la alusión a TD se remite únicamente a las didácticas. 
Alusión a TD en los programas de las asignaturas específicas por área

$38 \%$

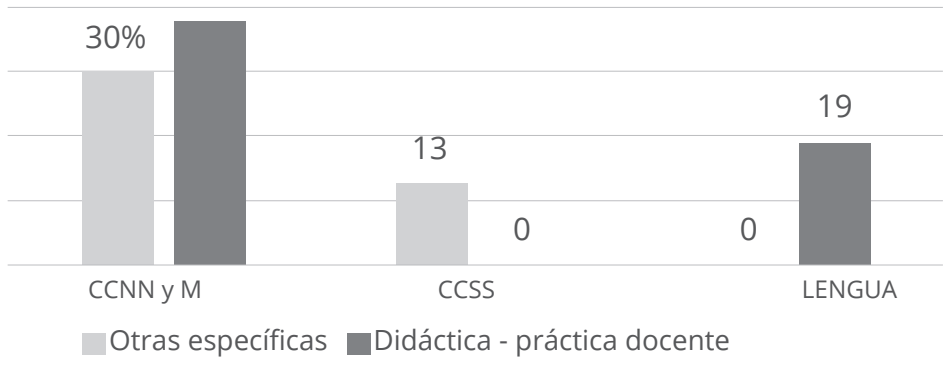

Figura $\mathbf{N}^{\circ}$ 8. Alusión a TD en los programas de las asignaturas específicas por área. Fuente: Elaboración propia.

En el área de CCNN y Matemática, además de resaltar la mayor presencia de referencias a las TD, es preciso mencionar que los valores más altos se encuentran en los profesorados de Matemática y Biología. Mientras que en el primero la mayor recurrencia se da en los programas de Didáctica (75\%), en el segundo el mayor porcentaje se da en los programas de otras asignaturas específicas (Figura 9).

Alusión a TD en los programas de las asignaturas específicas de Ciencias de la Naturaleza y Matemática

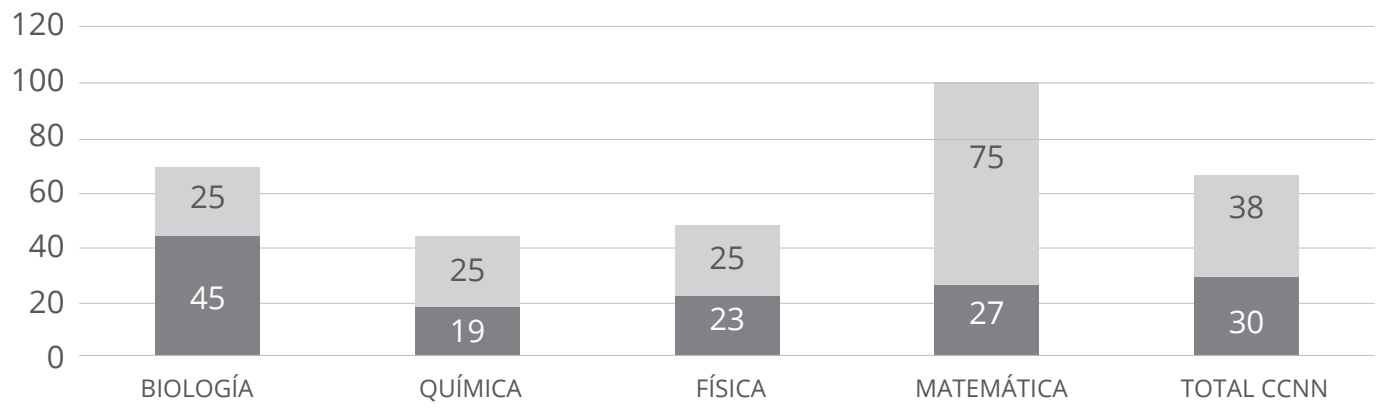

Otras específicas que aluden a TD Didáctica - práctica docente (\%)

Figura $\mathbf{N}^{\circ}$. Alusión a TD en los programas de las asignaturas específicas de CCNN y Matemática. Fuente: Elaboración propia.

\section{La asignatura Informática y su aporte a la formación tecnológica del futuro docente}

\section{Contenidos que se abordan en cada plan: lo instrumental versus lo didáctico}

La inclusión de tecnologías en los planes de FID analizados, además de aparecer como parte de los contenidos de las asignaturas de los diferentes programas ocupa un lugar de privilegio en la asignatura Informática. De acuerdo con lo expresado por Gewerc (2015, p. 42), cabe reflexionar en qué medida la propuesta de formación se remite a un abordaje instrumental o también proporciona las herramientas necesarias para que los futuros docentes se apropien de los recursos tecnológicos para aplicarlos en la práctica docente en pro de la mejora de la enseñanza. El análisis comparativo de los planes 2008 
y 2005 pone de manifiesto que, porcentualmente, el Plan 2008 dedica más unidades a un abordaje instrumental y didáctico que las que propone el Plan 2005 (Figura N 10). Si bien este aspecto puede ser interpretado como una mejora, es necesario mencionar aquí que el peso que se le da a la aplicación en la asignatura Informática no está acompañado por un abordaje de similares características desde los programas de Didáctica (20\%); asignatura que por excelencia se ocupa de preparar al futuro docente para su desempeño como profesor de educación media.

Tipo de contenidos: instrumental, aplicado y mixto

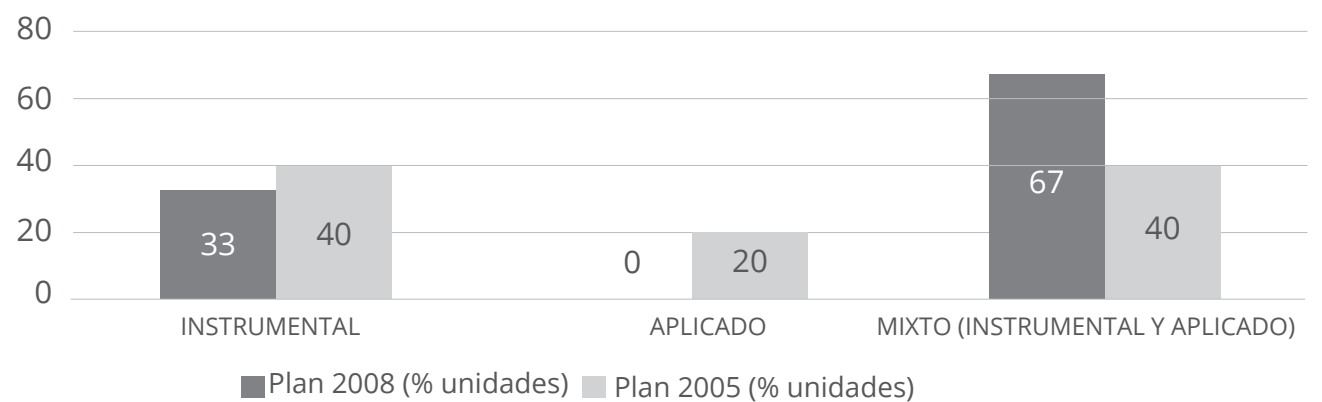

Figura $\mathbf{N}^{\circ}$ 10. Tipo de contenidos presentes en la asignatura Informática en los Planes 2005 y 2008. Fuente: Elaboración propia.

\section{Desarrollo de CD en la asignatura Informática}

Al momento de referirnos a la CD resulta necesario aclarar que no existe ninguna mención explícita ni al término competencia ni al término competencia digital. Ni siquiera aparece el término digital en ninguno de los dos planes analizados (Plan 2005 y Plan 2008). Dada la relevancia actual de este concepto en la formación de los docentes se recurrió al mismo como marco de análisis. Así, a partir de la lectura de los contenidos del programa de Informática se procuró identificar lo que cada contenido podría aportar a cada área de competencia, o competencia relacionada, con base en la clasificación propuesta por Ferrari (2013, p. 11). El cuadro de la Figura N 11 ofrece ejemplos de los pasajes de los programas del Plan 2008 que fueron categorizados dentro de cada subtipo identificado. Cabe señalar que en ambos planes solo se encontraron contenidos que se corresponden con el desarrollo de tres áreas de competencias de las ofrecidas por Ferrari (2013), a saber: informacional, comunicacional y creación de contenidos.

\begin{tabular}{|l|l|l|l|}
\hline $\begin{array}{l}\text { Plan 2008: } \\
\text { Programa de } \\
\text { Informática }\end{array}$ & \multicolumn{2}{|l|}{ Áreas de competencia digital } \\
\hline & Informacional & Comunicacional & Creación de contenidos \\
\hline $\begin{array}{l}\text { Conceptualización } \\
\text { (MCCDD: INTEF, } \\
\text { 2017.pp 15:46) }\end{array}$ & $\begin{array}{l}\text { Buscary acceder a } \\
\text { información en línea } \\
\text { para: encontrarla, } \\
\text { seleccionarla, } \\
\text { articularla, validarla y } \\
\text { retenerla. }\end{array}$ & $\begin{array}{l}\text { Utilizar las TD para: interactuar, } \\
\text { comprender las formas } \\
\text { adecuadas de comunicación } \\
\text { y cómo las mismas se } \\
\text { distribuyen, representan, } \\
\text { administran y adaptan a } \\
\text { audiencias específicas. }\end{array}$ & $\begin{array}{l}\text { Crear, editar y reelaborar } \\
\text { contenidos digitales } \\
\text { multimedia y programar. } \\
\text { Conocer y aplicar los } \\
\text { derechos de autor y } \\
\text { licencias de uso. }\end{array}$ \\
\hline $\begin{array}{l}\text { Evidencias que } \\
\text { ejemplifican }\end{array}$ & $\begin{array}{l}\text { "Búsqueda de } \\
\text { información en } \\
\text { Internet" (p.2). }\end{array}$ & $\begin{array}{l}\text { "Sitios destinados a impartir } \\
\text { cursos en aulas virtuales" (p.2). }\end{array}$ & $\begin{array}{l}\text { "Generación de contenidos } \\
\text { para el aula" (p.2). }\end{array}$ \\
\hline
\end{tabular}

Figura $\mathbf{N}^{\circ}$ 11. Ejemplos de contenidos del programa de Informática categorizados por su aporte al desarrollo de cada área de competencia digital. Fuente: Elaboración propia. 
Al momento de comparar la cantidad de contenidos correspondientes a cada área de competencia en cada plan es posible apreciar que en el Plan 2008 existe una distribución equitativa de los tres tipos mencionados, en tanto que en el Plan 2005 cobran mayor relevancia la competencia digital comunicacional y la de creación de contenidos (Figura $\mathrm{N}^{\circ}$ 12). Tales diferencias entre ambos planes pueden interpretarse como una disminución del protagonismo otorgado al estudiante dado que en el Plan 2008 existen menos ejemplos de contenidos concretos que estimulen la producción referida a recursos tecnológicos.

Competencias que se promueven desde

la asignatura informática de cada Plan

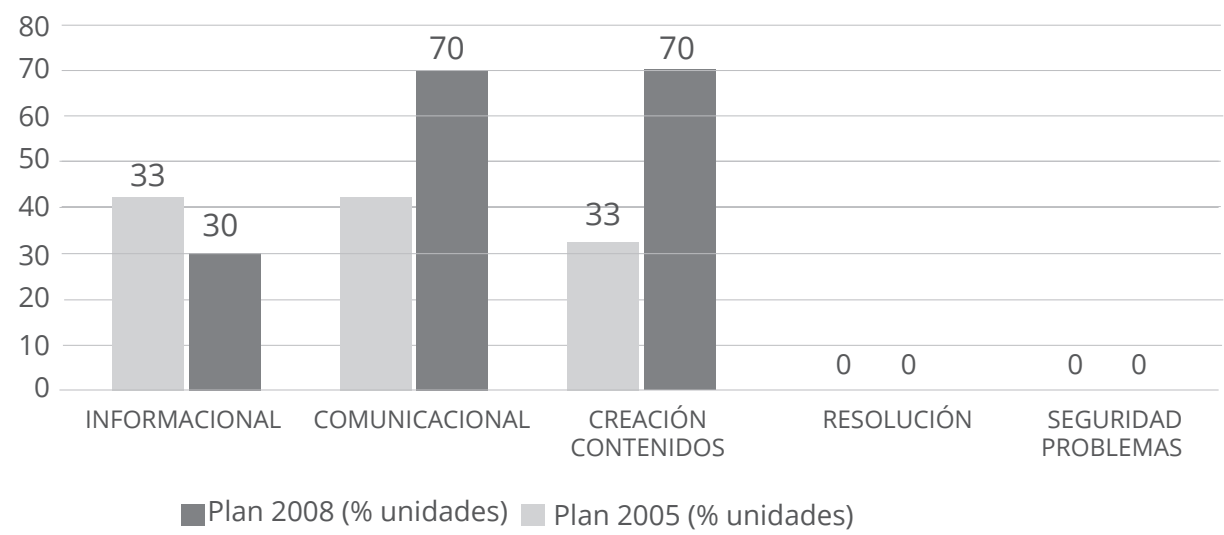

Figura $\mathbf{N}^{\circ}$ 12. Competencias que se promueven desde la asignatura Informática en cada Plan. Fuente: Elaboración propia.

\section{Conclusiones}

El proceso de indagación realizado procuró identificar presencias y ausencias en los programas de FID en referencia a la inclusión de TD. Las evidencias recabadas muestran que, en varios aspectos, el Plan actual (2008) constituye un retroceso en comparación con el Plan 2005 de profesorado. A continuación se detallan los principales hallazgos que permiten constatar que el cambio de Plan no refleja que existan mejoras en la incorporación y aprovechamiento de las TD.

En primer lugar corresponde señalar que al analizar la fundamentación de cada plan se encuentra que, por un lado, en el Plan 2008 se hace referencia de forma muy general a la tecnología, mientras que en el Plan 2005 se enfatiza en la relevancia de las TD aludiendo al impacto de las mismas en la formación instrumental y en el futuro desempeño profesional. Otro aspecto a destacar es que el cambio del Plan 2005 al Plan 2008 no tuvo criterios comunes en lo que refiere al lugar otorgado a las TD, ni para los diversos profesorados ni para las distintas asignaturas dentro de cada profesorado. Mientras que el Plan 2005 apunta a un abordaje de las TD focalizado en tres cursos de la asignatura Informática, cuyos contenidos marcan explícitamente la necesidad de establecer relaciones con las otras asignaturas, en el Plan 2008, aunque se dedican más unidades a un abordaje instrumental y didáctico disminuye la carga horaria de Informática. Asimismo, el plan vigente aumenta la alusión a las TD desde otras asignaturas, aunque ese aumento no es igual para los diferentes profesorados. En algunas especialidades aumentan las referencias a TD en las didácticas, y en otras el aumento se da en las otras asignaturas específicas. Cabe mencionar sin embargo, que en términos generales resultan escasas las referencias al trabajo con TD tanto en la asignatura Didáctica de los diferentes profesorados como en las otras asignaturas específicas, con una mayor presencia en el caso del área de 
CCNN y Matemática. Se constata además que existen pocos ejemplos de programas que hacen explícita la necesidad de relación entre el abordaje disciplinar, el didáctico y el tecnológico. De la lectura de todos los programas del Plan 2008 no surge la existencia de un posicionamiento claro respecto a si se pretende un abordaje puntual de las TD (desde el espacio de una asignatura) o si las mismas se abordan con carácter transversal.

En otro orden, del análisis realizado es posible apreciar que el Plan 2008, vigente en la actualidad, no se enmarca en el paradigma de competencias y, por tanto, son escasas las menciones que pueden interpretarse como un aporte al desarrollo genuino de la CD. Al momento de profundizar dicho análisis e intentar dilucidar qué áreas de la CD se traslucen de la propuesta de contenidos de la asignatura Informática, se constató que en el Plan 2005 cobra mayor relevancia el área de creación de contenidos, aspecto que se reduce sustancialmente en el Plan 2008. Esto puede interpretarse como una disminución del protagonismo otorgado al estudiante.

Luego de analizar las propuestas programáticas, y a la luz de las sugerencias teóricas consultadas se considera imprescindible que frente a la reformulación curricular futura se tengan en cuenta las potencialidades de las propuestas anteriores y se tomen decisiones que posibiliten la inclusión genuina de las TD en la formación de los educadores. Es en este sentido que se considera fundamental que la actualización de las propuestas de formación esté en sintonía con los nuevos enfoques. Es preciso además que se prevea una flexibilidad suficiente que permita hacer frente a los cambios tecnológicos permanentes (Avello et al., 2014). Del análisis se desprende asimismo la necesidad de incluir un abordaje específico de las TD en espacios curriculares propios pero que además se garantice la transversalidad desde todas las asignaturas del currículo. La transformación exige pues un compromiso de todos los involucrados en la formación, y ese compromiso comienza en el diseño de los planes de formación para lograr aproximarse a lo propuesto por Tondeur et al. (2016) cuando expresan que:

“Las instituciones de formación de docentes necesitan ayudar activamente a los futuros docentes a hacer las conexiones entre tecnología, pedagogía y conocimiento del contenido en todos los aspectos de su educación y campo de trabajo". (Tondeur et al., 2016, p.24, traducción propia).

\section{Referencias bibliográficas}

Avello, R. et al. (2014). Experiencia cubana sobre la formación del docente latinoamericano en tecnologías para la educación. Educación Media Superior 28 (3): 587-591 Disponible en: http://scieloprueba.sld.cu/pdf/ems/v28n3/ems17314.pdf

Belando-Montoro, M. (2014). Formación permanente del profesorado. Algunos recursos TIC para la docencia universitaria. Revista Iberoamericana de Educación, (V 65), 1-11. Disponible en: https://rieoei.org/RIE/article/view/324

Castañeda, L., Esteve, F. \& Adell, J. (2018) ¿Por qué es necesario repensar la competencia docente para el mundo digital? RED. Revista de Educación a Distancia. Núm. 56, Art. 6, 31 01-2018. Disponible en: http://www.um.es/ead/red/56/castaneda_et_al.pdf

Cejas, R., Navío, A. \& Barroso, J. (2016). Las competencias del profesorado universitario desde el modelo TPACK (conocimiento tecnológico y pedagógico del contenido). PíxelBit Revista de Medios y Educación, Vol 49. 105-119. Disponible en: www.redalyc.org/ pdf/368/36846509008.pdf

Cobo, C. (2016). La innovación pendiente. Reflexiones (y provocaciones) sobre educación, tecnología y conocimiento. Colección Fundación Ceibal. Debate: Montevideo. 
Consejo de la Unión Europea (2014). Conclusiones del Consejo, de 20 de mayo de 2014, sobre formación eficaz de los docentes. Diario Oficial de la Unión Europea. Disponible en: http://eur-lex.europa.eu/legal-content/ES/TXT/PDF/?uri=CELEX:52014XG0614(05)\&from=ES

Díaz Barriga Arceo, F. (2010). Los profesores ante las innovaciones curriculares. Revista iberoamericana de educación superior, 1(1), 37-57. Disponible en: http://www.scielo.org. $\mathrm{mx} / \mathrm{pdf} / \mathrm{ries} / \mathrm{v} 1 \mathrm{n} 1 / \mathrm{v} 1 \mathrm{n} 1 \mathrm{a} 4 . \mathrm{pdf}$

Durán Cuartero, M., Gutiérrez Porlán, I. \& Prendes Espinosa, M. P. (2016). Certificación de la competencia TIC del profesorado universitario: Diseño y validación de un instrumento. Revista mexicana de investigación educativa, 21(69), 527-556. Disponible en: http://www. scielo.org.mx/pdf/rmie/v21n69/1405-6666-rmie-21-69-00527.pdf

Esteve, F. \& Gisbert, M. (2011) El nuevo paradigma de aprendizaje y las nuevas tecnologías. Revista de Docencia Universitaria, 9(3), 55-73. Disponible en http://red-u.net/redu/files/ journals/1/articles/301/public/301-626-1-PB.pdf

Esteve, F. \& Parejo, J. (2014). La integración de las TIC en la formación docente: TPACK y competencia digital. Disponible en: https://www.researchgate.net/profile/Francesc_Esteve/ publication/233721559_La_integracion_de_las_TIC_en_la_formacion_docente_TPACK_y competencia_digital/links/02bfe5118d3f9c863b000000/La-integracion-de-las-TIC-en-laformacion-docente-TPACK-y-competencia-digital.pdf

Ferrari, A. (2013). DIGCOMP: A framework for developing and understanding digital competence in Europe. Yves Punie y Barbara N. Brečko (Eds.), Sevilla, España. Disponible en: http:// publications.jrc.ec.europa.eu/repository/bitstream/JRC83167/lb-na-26035-enn.pdf

Fullan, M. (2002). Las fuerzas del cambio (Vol. 5). Ediciones AKAL.

Gewerc, A. \& Alonso, A. (2017). Influencia del plan de estudios del grado de maestro de primaria en el conocimiento profesional del profesorado. El caso de la universidad de Santiago de Compostela. Revista del currículum y formación del profesorado., 21. Recuperado de https://dialnet.unirioja.es/servlet/articulo?codigo=6048318

Gewerc, A. \& Montero, L. (2013). Culturas, formación y desarrollo profesional. La integración de las TIC en las instituciones educativas. Revista de Educación, (362). Disponible en: https:// doi.org/10.4438/1988-592X-RE-2011-362-163

Gewerc, A. \& Montero, L. (2015). Conocimiento profesional y competencia digital en la formación del profesorado. El caso del Grado de Maestro en Educación Primaria. RELATEC - Revista Latinoamericana de Tecnología Educativa, (V. 14, n. 1), 31-43. Disponible en: https:// doi.org/10.17398/1695-288X.14.1.31

Gros, B. (2000). El ordenador invisible: hacia la apropiación del ordenador en la enseñanza. Barcelona: Gedisa.

Gros, B. y Guerrero, C. (Eds.) (2016). Pedagogía red. Una educación para tiempos de Internet. Barcelona: Ediciones OCTAEDRO-ICE. Disponible en: https://www.octaedro.com/appl/ botiga/client/img/15202.pdf

Gülbahar, Y. (2008). ICT usage in higher education: a case study on preservice teachers and instructors. TOJET: Turkish Online Journal of Educational Technology, 7(1), 32-37. 
Hernández Sampieri, R., Fernández Collado, C. y Baptista Lucio, P. (2014). Metodología de la Investigación (5a edición). México. Mc. Graw Hill.

INTEF (2017). Marco Común de Competencia Digital Docente - Setiembre 2017. Disponible en: https://aprende.intef.es/sites/default/files/2018-05/2017_1020_Marco-Com\%C3\%BAn-deCompetencia-Digital-Docente.pdf

Koehler, M. \& Mishra, P. (2009). What is technological pedagogical content knowledge? Contemporary Issues in Technology and Teacher Education, 9 (1) 60-70. Disponible en: https://citejournal.s3.amazonaws.com/wp-content/uploads/2016/04/v9i1general1.pdf

Levis, D. (2016). Enseñar y aprender en la sociedad de la Pantalla: hacia la escuela tecnómada. Actas de Periodismo y Comunicación, Vol. 2, N. ${ }^{\circ}$. Universidad Nacional de La Plata. La Plata-Buenos Aires-Argentina. Disponible en: http://sedici.unlp.edu.ar/bitstream/ handle/10915/60940/Documento_completo.pdf-PDFA.pdf?sequence=1

Lion, C. (2015). Desarrollos y tejidos actuales en el campo de la tecnología educativa: caleidoscopio en movimiento. Archivos de Ciencias de la Educación (V 9) 1-13. Disponible en: http://www.memoria.fahce.unlp.edu.ar/art_revistas/pr.7037/pr.7037.pdf

Marcelo, C. (1995). Formación del profesorado para el cambio educativo. Barcelona, EUB.

Montero, L. (2001). La construcción del conocimiento profesional docente. Rosario, Argentina: Homo Sapiens.

Ottenbreit-Leftwich, A. et al. (2012). Preparation versus practice: How do teacher education programs and practicing teachers align in their use of technology to support teaching and learning? Computers \& Education 59, 399-411.

Ottestad, G., Gudmundsdottir, G. y Kelentric, M. (2014). Professional digital competence in teacher education. Nordic Journal of Digital Literacy.

Perrenoud, P. (2007). Diez nuevas competencias para enseñar. Gráficas Monte Albán. México. Disponible en: https://www.uv.mx/dgdaie/files/2013/09/Philippe-Perrenoud-Diez-nuevascompetencias-para-ensenar.pdf

Quiroga, L. (2011). Posibilidades y limitaciones de las tecnologías de la información y la comunicación (TIC) para la docencia. Actualidades pedagógicas. (V 58) 65-79. Disponible en: https://revistas.lasalle.edu.co/index.php/ap/article/view/539

Robalino, M. \& Körner, A. (2005) Experiencias de formación docente utilizando tecnologías de información y comunicación. Estudios realizados en Bolivia, Chile, Colombia, Ecuador, México, Panamá, Paraguay y Perú. Unesco. Disponible en: http://unesdoc.unesco.org/ images/0014/001410/141010s.pdf

Rodríguez Zidán, E., Marcelo, C., Yot, C., Zorrilla Salgador, J.P. y Cabrera, C. (2018). Determinantes y usabilidad de los ordenadores portátiles en la formación inicial de profesorado en Uruguay. Documento de trabajo WP04-05/18, Instituto de Educación, Universidad ORT Uruguay. Disponible en: https://ie.ort.edu.uy/innovaportal/file/71429/1/ determinantes-y-usabilidad-de-los-ordenadores-portatiles.pdf 
Siddiq, F., Scherer, R. \& Tondeur, J. (2016). Teachers' emphasis on developing students' digital information and communication skills (TEDDICS): A new construct in 21 st century education. Computers \& Education, 92, 1-14.

Silva Quiroz, J., Miranda, P. \& Gisbert, M. (2016). Indicadores para evaluar la competencia digital docente en la formación inicial en el contexto Chileno-Uruguayo. RELATEC Revista Latinoamericana de Tecnología Educativa, (V. 15), 55-67. Disponible en: https://doi. org/10.17398/1695-288X.15.3.55

Tondeur, J., Pareja, N., Braak, J., Voogt, J. \& Prestridge, S. (2016). Preparing beginning teachers for technology integration in education: ready for take-off?

Computers \& Education, Art. 26. Disponible en: https://www.researchgate.net/ publication/289533333

Tondeur, J., Aesaert, K., Prestridge, S. \& Consuegra, E. (2018). A multilevel analysis of what matters in the training of pre-service teacher's ICT competencies. Computers \& Education, 56, Art. 6. Disponible en: https://doi.org/10.1016/j.compedu.2018.03.002

Unesco (2004). Las tecnologías de la información y la comunicación en la formación docente. Guía de planificación. Montevideo-Uruguay. Editorial Trilce Disponible en: http://unesdoc. unesco.org/images/0012/001295/129533s.pdf

Unesco (2011). Unesco ICT Competency framework for teachers. Version 2.0. París: Ediciones de la Organización de las Naciones Unidas para la Educación, la Ciencia y la Cultura; 2011. Disponible en: http://unesdoc.unesco.org/images/0021/002134/213475E.pdf

Unesco (2016). Revisión comparativa de iniciativas nacionales de aprendizaje móvil en América Latina. El caso de la Política TIC en Perú. Organización de las Naciones Unidas para la Educación, la Ciencia y la Cultura. Disponible en: http://repositorio.minedu. gob.pe/bitstream/handle/123456789/4543/Revisi\%C3\%B3n\%20comparativa\%20 de $\% 20$ iniciativas $\% 20$ nacionales $\% 20$ de $\% 20$ aprendizaje $\% 20 \mathrm{~m} \%$ C3\%B3vil\%20en $\% 20$ Am\%C3\%A9rica\%20Latina\%20el\%20caso\%20de\%20la\%20pol\%C3\%ADtica\%20TIC\%20 en\%20el\%20Per\%C3\%BA.pdf?sequence=1\&isAllowed $=y$

Vaillant, D. (2013). Integración de TIC en los sistemas de formación docente inicial y continua para la Educación Básica en América Latina. Recuperado de: https://drive.google.com/ file/d/1lifv33K-2oWxwW11/8S2-ktgBrlhBp6y/edit

Valverde, J. (2015). La formación inicial del profesorado en el grado en Educación Primaria. Una valoración cualitativa del diseño y desarrollo curricular de la asignatura "Recursos tecnológicos, didácticos y de investigación". Tendencias pedagógicas (Vol. 25) pp. 207-228. Disponible en: https://dialnet.unirioja.es/descarga/articulo/5164826.pdf

Yuni, J. A. \& Urbano, C. A. (2006). Técnicas para investigar y formular proyectos de investigación. Córdoba, Argentina: Editorial Brujas.

\section{(Endnotes)}

${ }^{1}$ La presente comunicación se enmarca en el Proyecto PRAXIS: Formación pedagógicodidáctica en tecnologías y práctica docente (FSED_3_2016_1_133331) aprobado en la convocatoria del Fondo Sectorial de Educación y financiado por la Agencia Nacional de Investigación e Innovación de Uruguay (ANII) y el Consejo de Formación en Educación (CFE). 